REVIEW

\title{
Biochemical markers of myocyte injury in heart failure
}

\author{
Y Sato, T Kita, Y Takatsu, T Kimura
}

Heart 2004;90:1110-1113. doi: 10.1136/hrt.2003.023895

This review discusses the role of biochemical markers of myocyte injury in patients with chronic congestive heart failure. Heart specific assays have been developed for the measurement of cardiac troponin T (cTnT), cardiac troponin I (cTnl), heart type fatty acid binding protein $(\mathrm{H}-$ FABP), and myosin light chain 1 (MLC-1). Concentrations of these biochemical markers increase in the absence of ischaemic events in the subset of patients with heart failure whose long term outcomes are most adverse. The markers are easy to measure serially and it is therefore easy to follow patients without inter-observer variability. The serial clinical use of these markers, separately or in combination, will sharpen our understanding of the state of heart failure.

See end of article for authors' affiliations

\section{Correspondence to:} Dr Y Sato, Department of Cardiovascular Medicine, Graduate School of Medicine, Kyoto

University, 54 Kawaracho Shogoin, Sakyo-ku, Kyoto 606-8507, Japan; cardioys@kuhp.kyoto-u. ac.jp

Accepted 5 February 2004
C hronic congestive heart failure is associated with a dismal long term prognosis and remains a major worldwide health concern. While various management strategies have become available, clinical tools to stage chronic heart failure remain few. New York Heart Association (NYHA) functional classification, along with several tests, including chest $x$ ray, echocardiogram, myocardial scintigraphy, cardiopulmonary exercise, and haemodynamic measurements are useful to estimate the degree of heart failure, though are subject to interobserver variations in interpretation. ${ }^{12}$

The loss of cardiac function in patients with chronic heart failure may be caused by ventricular remodelling, a process by which ventricular size, shape, and function are altered by mechanical, neurohormonal, and genetic factors. Ventricular remodelling consists of myocyte hypertrophy and slippage, loss of myocytes, decrease in myofibril content, and myocardial interstitial fibrosis. ${ }^{3}$ Serial measurements of reliable biochemical markers of myocyte injury in patients with heart failure would be helpful to monitor their long term progress, without interobserver variability. This review discusses our understanding, and the significance, of current biochemical markers detected in patients with chronic heart failure.

\section{BIOCHEMICAL MARKERS OF MYOCYTE INJURY}

Cardiac enzymes have long been used as frontline diagnostic tools in the detection of myocardial injury caused by myocardial ischaemia. ${ }^{5}$ However, the most commonly used enzymes, including creatine kinase (CK) and its myocardial fraction CK myocardial band (MB), aspartate aminotransferase, and lactate dehydrogenase, are limited in their ability to detect myocardial injury by short diagnostic windows, limited sensitivities, and lack of specificity because of their presence in skeletal muscle. Furthermore, studies performed as long as two decades ago found no correlation between serum concentrations of $\mathrm{CK}$ or $\mathrm{CK}-\mathrm{MB}$ and heart failure. ${ }^{6}$ Myoglobin, an $18 \mathrm{kD}$ cytosolic protein, also lacks specificity because its release from skeletal muscle cannot be distinguished from its release from heart muscle. ${ }^{7}$

These limitations, as well as the known unique amino acid sequence of myofibrillar cardiac proteins, prompted the development of monoclonal antibodies for the detection of cardiac troponins by immunoassay. The subunits $\mathrm{I}, \mathrm{T}$, and $\mathrm{C}$ of the troponin complex on the actin filament regulate the force and velocity of muscle contraction. The $37 \mathrm{kD} \mathrm{T}$ subunit is responsible for binding the troponin complex to tropomyosin. The first generation of cTnT assays were flawed by spuriously increased values in patients with severe skeletal muscle or renal disorders, ${ }^{89}$ perhaps from cross-reactivity of the cTnT assay with skeletal muscle troponin $\mathrm{T}$, or the expression of cTnT by skeletal muscles during regenerative processes, particularly in patients with neuromuscular disorders and nephropathies. However, the latest cTnT assay is a sensitive and specific marker of myocyte injury, even in the presence of these disorders. ${ }^{10}{ }^{11}$

CTnI $(21 \mathrm{kD})$ prevents contraction in the absence of calcium by inhibiting the adenosine triphosphatase activity of the actin-myosin interaction. CTnI is highly cardiac specific because of the dissimilarity of a 31 amino acids sequence on the $\mathrm{N}$-terminus compared to that of skeletal troponins; it is also absent during human skeletal muscle regeneration. ${ }^{12}$ cTnT and cTnI are highly sensitive and specific markers of myocardial injury in acute coronary syndromes. In addition, increased serum concentrations of these markers have been associated with adverse short and long term outcomes in patients with unstable angina or acute myocardial infarction. $^{13}{ }^{14}$ Moreover, the background concentration of cTnT and cTnI is very low while the background level of CK, aspartate aminotransferase, and lactate dehydrogenase is significant even in normal conditions. CTnT and CTnI,

Abbreviations: BNP, brain natriuretic peptide; cTnl, cardiac troponin I; cTnT, cardiac troponin $\mathrm{T}$; CK, creatine kinase; DCM, dilated cardiomyopathy; H-FABP, heart type fatty acid binding protein; $M B$, myocardial band MLC-1, myosin light chain 1; NYHA, New York Heart Association 
therefore, have replaced CK-MB as the standard marker in acute coronary syndromes, and a new definition of acute myocardial infarction has been developed, based on increases in cardiac troponins in the blood. ${ }^{15}{ }^{16}$

The mechanisms of release and clearance of cTnT and cTnI are incompletely understood. Although both are structural proteins, it has been suggested that cytosolic pools of these proteins are released into the circulation after cell injury. The cytosolic pool for cTnT was estimated at $6-8 \%,{ }^{13}$ and that for soluble cTnI at $2.8 \%{ }^{14}$ The release of cTnT in ischaemic myocardial injury may be because of transient leakage from the cytosolic component from loss of sarcolemmal integrity during reversible ischaemia, ${ }^{17}$ or from its continuous release when ischaemic injury is irreversible. ${ }^{18}$

Other biochemical markers of myocyte injury have also been described. H-FABP, a $15 \mathrm{kD}$ cytoplasmic protein involved in lipid homeostasis, is abundant in heart muscle. ${ }^{19}{ }^{20}$ It has recently been reported to detect early myocyte injury in patients with acute myocardial infarction. ${ }^{21}$ Myosin is a structural protein of the sarcomere; a heart specific assay using monoclonal antibodies against MLC-1 has been previously described in patients with acute myocardial infarction. ${ }^{22}$

\section{BIOCHEMICAL MARKERS OF MYOCYTE INJURY IN PATIENTS WITH HEART FAILURE \\ CTnT}

Congestive heart failure is a clinical syndrome which may develop from a variety of diseases. Dilated cardiomyopathy (DCM) is a primary myocardial disorder of unknown aetiology characterised by ventricular dilatation and depressed myocardial contractility, which leads to chronic heart failure without apparent myocardial ischaemia. While ongoing myocyte injury has been documented by ${ }^{111}$ indium anti-myosin antibody imaging in patients with $\mathrm{DCM}^{23}$ this technique requires radioisotopes and cannot be used to follow patients serially in the long term.

In an earlier study, persistently high serum concentrations of cTnT were observed over several years of follow up in approximately $30 \%$ of our DCM patients. ${ }^{24}{ }^{25}$ These patients had a significantly greater decrease in left ventricular ejection fraction and higher rates of long term adverse outcomes than patients without increased cTnT concentrations. It is particularly noteworthy that, in most patients, cTnT concentrations remained elevated after the patients were stabilised clinically by conventional therapy, free of dyspnoea, and without radiographic and auscultatory signs of pulmonary congestion. These observations indicate that cTnT is a marker of subclinical myocyte injury even when heart failure is compensated. In that study of patients with DCM, we chose $0.02 \mathrm{ng} / \mathrm{ml}$ as the upper normal limit of serum concentration, a relatively low value compared with patients with ischaemic heart disease.

Cardiomyopathic disorders are associated with predominantly systolic or diastolic dysfunction, or with both. In hypertrophic cardiomyopathy, which is initially associated with predominant diastolic dysfunction, ${ }^{26}$ we recently reported increased concentrations of cTnT in $50 \%$ of patients during the non-dilated phase of the disease, when systolic function was preserved, and in the absence of ischaemia. ${ }^{27}$ Some patients had increased cTnT concentrations persisting over several years of follow up, during which fractional shortening and intraventricular septum thickness decreased significantly. These observations indicate that cTnT is a marker of myocyte injury in patients with hypertrophic cardiomyopathy. In a univariate analysis, Dispenzieri and colleagues found cTnT, cTnI, septal thickness, left ventricular ejection fraction, urine $M$ spike, age, and symptoms of congestive heart failure to be significant predictors of overall survival in patients with cardiac amyloidosis, while in multivariate analysis, the detection of cTnT was the most reliable predictor. ${ }^{28}$

Though we initially studied the significance of CTnT as a marker of myocyte injury in patients with heart failure and non-ischaemic disorders, ischaemic heart disease remains the predominant cause of chronic heart failure. We and others have reported the presence of increased cTnT concentrations in patients with heart failure, old myocardial infarctions, and without ongoing ischaemic events. ${ }^{29}{ }^{30}$ Ventricular remodelling after myocardial infarction may occur over several weeks or months, while other factors may also contribute to the progression of left ventricular dysfunction, including recurrent ischaemia with myocardial stunning, hibernation of the myocardium caused by a sustained reduction in myocardial blood flow, and the vascular and myocardial effects of endothelial dysfunction. ${ }^{4}$

While more studies of cTnT during cardiac remodelling after myocardial infarction should further promote its acceptance as a monitoring tool in patients with heart failure, the interpretation of results in patients with ischaemic heart disease is not without ambiguity. Since patients with heart failure after a healed myocardial infarction may have asymptomatic stenoses of one or more, large or small, coronary arteries, increased concentrations of markers of myocyte injury may be a manifestation of ischaemic myocardium in the territory of the stenotic artery. Therefore, patients with chronic heart failure after an old myocardial infarction may have to undergo serial coronary angiograms to clarify the mechanism of cTnT release. ${ }^{31}$

We have collected preliminary data in patients with old myocardial infarctions who have undergone coronary revascularisation, and had persistently elevated CTnT and progression of heart failure, in the absence of increased CK or ischaemic events (unpublished data). Although cTnT is usually elevated in patients with unstable angina or acute coronary syndrome, ${ }^{32}{ }^{33}$ the persistently high concentrations, measured for several months or years after myocardial infarction, in our patients with chronic heart failure could not be attributed to unstable angina. Further studies are needed to clarify the significance of persistently elevated cTnT in the process of chronic ventricular remodelling after myocardial infarction.

Valvar and congenital heart diseases are other major causes of chronic heart failure, which may progress despite successful surgical interventions. ${ }^{34}$ We and others have found that some patients with valvar and congenital diseases also have elevated cTnT concentrations in the absence of cardiac ischaemia. ${ }^{29} 3035$ Our preliminary observations of persistently high cTnT concentrations in some patients after surgical repair may be useful for the post-operative monitoring of chronic myocyte injury (unpublished data).

\section{cTnl}

The first description of a biochemical marker of myocyte injury in patients with heart failure was offered in 1995 by Missov and colleagues, who reported increased cTnI concentrations in patients with NYHA class III and IV heart failure caused by DCM, or secondary to ischaemic disease. ${ }^{36}{ }^{37}$ Cardinale and associates reported that the elevation of cTnI in patients treated with high doses of chemotherapeutic agents for aggressive malignancies predicted the subsequent evolution of left ventricular function..$^{38}$ They concluded that cTnI is a sensitive and reliable marker of myocardial injury caused by high dose chemotherapy. Schultz and colleagues reported an exercise induced increase in cTnI concentrations in patients with heart failure, though the prognostic value of this finding should be further investigated. ${ }^{39}$ 
A recent study evaluated 238 advanced heart failure patients referred for cardiac transplantation evaluation who had a cTnI assay drawn at the time of initial presentation. Patients with acute myocardial infarction were excluded. Detectable cTnI was associated with progressive decline in ejection fraction over time and with increased mortality. Patients with ischaemic and non-ischaemic causes of heart failure were similar in terms of cTnI concentrations and other laboratory values, NYHA class, left ventricular ejection fraction, and haemodynamics. ${ }^{40}$

\section{H-FABP}

Recently, Setsuta and colleagues, who previously reported the elevation of CTnT in patients with heart failure, ${ }^{30}$ reported that H-FABP was associated with subsequent cardiac events in patients with chronic heart failure caused by DCM, old myocardial infarction, hypertensive heart disease, valvar heart disease, or congenital heart disease. ${ }^{41}$ While both cTnT and H-FABP were associated with subsequent cardiac deaths or rehospitalisation for the management of worsening heart failure, H-FABP was much more detectable among patients in NYHA functional class II. H-FABP is a small protein abundant in the cytosol which is readily released into the circulation following myocardial damage. In contrast, most troponins are components of the myofibrillar contractile apparatus, present in small amounts in the cytosol. This may explain the different patterns of increase of these two markers following myocyte injury.

\section{MLC- 1}

Studies of MLC-1, a $27 \mathrm{kD}$ protein, as a biochemical marker of myocyte injury in patients with heart failure are few. Hansen and colleagues reported that circulating MLC-1 was elevated in some patients in NYHA functional class III and IV, and this increase was associated with a poor prognosis in a clinical trial of flosequinin. ${ }^{42}$ Studies are needed to further characterise this marker in patients with heart failure and to distinguish it from the other biochemical markers of myocyte injury described earlier.

\section{USE OF BIOCHEMICAL MARKERS TO MEASURE THE DEGREE OF HEART FAILURE}

Since heart failure is a complex clinical syndrome, a single biomarker may not reflect all of its characteristics. The serial and combined measurements of biochemical markers of myocyte injury may open new perspectives in heart failure. Brain natriuretic peptide (BNP) is an amino acid peptide chiefly secreted by the ventricular myocardium in response to strain. The plasma measurement of BNP is being used increasingly in the diagnosis, prognosis, and monitoring of patients with congestive heart failure. ${ }^{43-45}$ BNP may be viewed as a marker of myocardial load and cTnT as a marker of myocyte injury. Combining these biochemical markers may provide new insight in the management of heart failure. In our small study of patients presenting with decompensated heart failure, approximately one third had initial concentrations of cTnT within normal limits. While BNP decreased significantly after treatment in all patients, cTnT remained elevated in most patients whose initial concentrations were increased, despite radiographic resolution of pulmonary congestion. ${ }^{29}$ We hypothesise that a first therapeutic goal should consist of relief of circulatory congestion and lowering of BNP, and a second goal be the mitigation of myocyte injury and lowering of cTnT. Recently, Ishii and colleagues reported that cTnT concentrations $>0.033 \mathrm{ng} / \mathrm{ml}$ and BNP concentrations $>440 \mathrm{pg} / \mathrm{ml}$, at the time of admission to the coronary care unit, correlated with significantly higher rates of cardiac events among 98 consecutive patients hospitalised for management of worsening chronic heart failure. ${ }^{35}$
At this time, the relative contributions of cTnT, cTnI, H-FABP, and MLC-1 in patients with heart failure remain unclear. The different half-lives, molecular sizes, and intracellular distributions of these markers may provide detailed information regarding the process of myocyte injury by monitoring the markers in combination. Combinations of markers of myocyte injury and markers of interstitial matrix collagen turnover may also add new information on the process of cardiac remodelling in patients with chronic heart failure. ${ }^{24}$

\section{FUTURE APPLICATIONS OF BIOCHEMICAL MARKERS IN HEART FAILURE Mechanisms of myocyte injury and biochemical markers}

Although these biochemical markers indicate the presence of ongoing myocyte injury in patients with heart failure, the mechanisms of that injury remain unclear. In our study of DCM, the presence of active myocarditis was excluded by endomyocardial biopsies using the Dallas criteria. ${ }^{25}$ Furthermore, transverse sections of postmortem cardiac specimens from three patients with DCM with persistently elevated cTnT showed no significant mononuclear cellular infiltration (unpublished data). The mechanism of myocyte injury without cellular infiltration needs to be studied. Adrenergic stimulation, calcium handling abnormalities, the reninangiotensin system, endothelin, inflammatory cytokines, nitric oxide, oxidative stress, and mechanical stress have been explored as potential contributors to myocyte injury in the setting of heart failure. ${ }^{46}{ }^{47}$ The existence of correlations among these factors with biochemical markers of myocyte injury should be examined in clinical studies to provide important information applicable to the management of heart failure.

\section{Biochemical markers as surrogate end points in heart failure}

Since heart failure is a life threatening condition, survival was chosen as the primary end point in the clinical trials which proved the effectiveness of angiotensin-converting enzyme inhibitors, aldosterone antagonists, and $\beta$ adrenergic blockers ${ }^{48}$ However, large study populations and long study periods are usually required to show a significant effect of treatment on survival. Therefore, the interest in surrogate end points has recently increased, since their use may allow the successful completion of controlled clinical trials with smaller patient populations, within shorter observation periods. Combinations of certain biochemical markers described earlier may represent surrogate endpoints suitable for the design of such trials. ${ }^{49}$

\section{CONCLUSIONS}

No guidelines have been issued regarding the monitoring of biochemical markers of myocyte injury as part of the management of chronic heart failure. Recent technological advances will allow the rapid application of these assays in the near future. The real time detection of myocyte injury will render the management of heart failure more precise and effective. It is our expectation that these assays will become the new standards in the monitoring of patients with heart failure.

\footnotetext{
Authors' affiliations

Y Sato, T Kita, T Kimura, Department of Cardiovascular Medicine, Graduate School of Medicine, Kyoto University, Kyoto, Japan Y Takatsu, Department of Cardiovascular Medicine, Amagasaki Hospital, Japan
} 


\section{REFERENCES}

1 Hunt SA, Baker DW, Chin MH, et al. ACC/AHA guidelines for the evaluation and management of chronic heart failure in the adult: executive summary. $J$ Heart Lung Transplant 2002;21:189-203.

2 Remme WJ, Swedberg K. Task force for the diagnosis and treatment of chronic heart failure, European Society of Cardiology. Guidelines for the diagnosis and treatment of chronic heart failure. Eur Heart $J$ 2001;22:1527-60.

3 Cohn JN, Ferrari R, Sharpe N. Cardiac remodeling-concepts and clinical implications: a consensus paper from an international forum on cardiac remodeling. On behalf of an international forum on cardiac remodeling. J Am Coll Cardiol 2000;35:569-82.

4 St John Sutton MG, Sharpe N. Left ventricular remodeling after myocardial infarction: pathophysiology and therapy. Circulation 2000;101:2981-8.

5 Crowley LV. Creatine phosphokinase activity in myocardial infarction, heart failure, and following various diagnostic and therapeutic procedures. Clin Chem 1968;14:1185-96.

6 Chemnitz G, Schmidt E, Schmidt FW, et al. Determination of creatine kinase and CK-MB in heart failure. Med Klin 1978;73:1809-11.

7 Christenson RH, Azzazy HM. Biochemical markers of the acute coronary syndromes. Clin Chem 1998;44:1855-64.

8 Bodor GS, Survant L, Voss EM, et al. Cardiac troponin T composition in normal and regenerating human skeletal muscle. Clin Chem 1997:43:476-84.

9 Bhayana V, Gongoulias T, Cohoe S, et al. Discordance between results for serum troponin T and troponin I in renal disease. Clin Chem 1995;41:312-7.

10 Baum H, Braun S, Gerhardt W, et al. Multicenter evaluation of a secondgeneration assay for cardiac troponin T. Clin Chem 1997:43:1877-84.

11 Ricchiuti V, Voss EM, Ney A, et al. Cardiac troponin T isoforms expressed in renal diseased skeletal muscle will not cause false-positive results by the second generation cardiac troponin T assay by Boehringer Mannheim. Clin Chem 1998;44:1919-24.

12 Bodor GS, Porterfield D, Voss EM, et al. Cardiac troponin I is not expressed in fetal and healthy or diseased adult human skeletal muscle tissue. Clin Chem 1995;41:1710-5

13 Katus HA, Remppis A, Neumann FJ, et al. Diagnostic efficiency of troponin T measurements in acute myocardial infarction. Circulation 1991:83:902-12.

14 Adams JE 3rd, Schechtman KB, Landt Y, et al. omparable detection of acute myocardial infarction by creatine kinase $M B$ isoenzyme and cardiac troponin I. Clin Chem 1994;40:1291-5.

15 Alpert JS, Thygesen K, Antman E, et al. Myocardial infarction redefined-a consensus document of the Joint European Society of Cardiology/American College of Cardiology Committee for the redefinition of myocardial infarction. J Am Coll Cardiol 2000;36:959-69.

16 Jaffe AS, Ravkilde J, Roberts R, et al. It's time for a change to a troponin standard. Circulation 2000;102:1216-20.

17 Piper HM, Schwarz P, Spahr R, et al. Early enzyme release from myocardial cells is not due to irreversible cell damage. J Mol Cell Cardiol 1984;16:385-8.

18 Katus HA, Remppis A, Scheffold T, et al. Intracellular compartmentation of cardiac troponin T and its release kinetics in patients with reperfused and nonreperfused myocardial infarction. Am J Cardiol 1991;67:1360-7.

19 Schaap FG, Van der Vusse GJ, Glatz JF. Fatty acid-binding proteins in the heart. Mol Cell Biochem 1998;180:43-51.

20 Glatz JF, Van Bilsen M, Paulussen RJ, et al. Release of fatty acid-binding protein from isolated rat heart subjected to ischemia and reperfusion or to the calcium paradox. Biochem Biophys Acta 1988;961:148-52.

21 Ishii J, Wang J, Naruse H, et al. Serum concentrations of myoglobin vs human heart-type cytoplasmic fatty acid-binding protein in early detection of acute myocardial infarction. Clin Chem 1997;43:1372-8.

22 Isobe M, Nagai R, Ueda S, et al. Quantitative relationship between left ventricular function and serum cardiac myosin light chain I level after coronary reperfusion in patients with acute myocardial infarction. Circulation 1987;76:1251-61.

23 Matsumori A, Kawai C, Yamada T, et al. Mechanism and significance of myocardial uptake of antimyosin antibody in myocarditis and cardiomyopathy: clinical and experimental studies. Clin Immunol Immunopathol 1993;68:215-9.
24 Sato Y, Kataoka K, Matsumori A, et al. Measuring serum aminoterminal type III procollagen peptide, $7 \mathrm{~S}$ domain of type IV collagen, and cardiac troponin T in patients with idiopathic dilated cardiomyopathy and secondary cardiomyopathy. Heart 1997;78:505-8.

25 Sato $Y$, Yamada T, Taniguchi $R$, et al. Persistently increased serum concentrations of cardiac troponin $\mathrm{T}$ in patients with idiopathic dilated cardiomyopathy are predictive of adverse outcomes. Circulation 2001;103:369-74.

26 European Study Group on Diastolic Heart Failure. How to diagnose diastolic heart failure. Eur Heart J 1998:19:990-1003.

27 Sato Y, Taniguchi R, Nagai K, et al. Measurements of cardiac troponin T in patients with hypertrophic cardiomyopathy. Heart 2003;89:659-60.

28 Dispenzieri A, Kyle RA, Gertz MA, et al. Survival in patients with primary systemic amyloidosis and raised serum cardiac troponins. Lancet 2003:361:1787-9.

29 Sato Y, Taniguchi R, Makiyama T, et al. Measurements of serum cardiac troponin $T$ and plasma brain natriuretic peptide in patients with severe cardiac decompensation. Heart 2002;88:647-8.

30 Setsuta K, Seino Y, Takahashi N, et al. Clinical significance of elevated levels of cardiac troponin T in patients with chronic heart failure. Am J Cardiol 1999;84:608-11

31 Sato $Y$, Taniguchi R, Yamada T, et al. Measurements of serum cardiac troponin T in patients with heart failure. Am Heart J 2003;145:e18.

32 Donnelly R, Millar-Craig MW. Cardiac troponins: IT upgrade for the heart. Lancet 1998;351:537-9.

33 Coudrey L. The troponins. Arch Intern Med 1998;158:1173-80.

34 Tornos MP, Olona M, Permanyer-Miralda G, et al. Heart failure after valve replacement for aortic regurgitation: prospective 20-year study. Am Heart J 1998:136:681-7.

35 Ishii J, Nomura M, Nakamura Y, et al. Risk stratification using a combination of cardiac troponin $T$ and brain natriuretic peptide in patients hospitalized for worsening chronic heart failure. Am J Cardiol 2002;89:691-5.

36 Missov E, Calzolari C. Elevated cardiac troponin I in some patients with severe congestive heart failure [abstract]. J Mol Cell Cardiol 1995;27:A-405.

37 Missov E, Calzolari C, Pau B. Circulating cardiac troponin I in severe congestive heart failure. Circulation 1997;96:2953-8

38 Cardinale D, Sandri MT, Martinoni A, et al. Left ventricular dysfunction predicted by early troponin I release after high-dose chemotherapy. J Am Coll Cardiol 2000;36:517-22

39 Schulz O, Kromer A. Cardiac troponin I: a potential marker of exercise intolerance in patients with moderate heart failure. Am Heart $J$ 2002; 144:351-8.

40 Horwich TB, Patel J, MacLellan WR, et al. Cardiac troponin I is associated with impaired hemodynamics, progressive left ventricular dysfunction, and increased mortality rates in advanced heart failure. Circulation 2003;108:833-8

41 Setsuta K, Seino Y, Ogawa T, et al. Use of cytosolic and myofibril markers in the detection of ongoing myocardial damage in patients with chronic heart failure. Am J Med 2002; 113:717-22.

42 Hansen MS, Stanton EB, Gawad Y, et al. Canadian PROFILE investigators. Relation of circulating cardiac myosin light chain 1 isoform in stable severe congestive heart failure to survival and treatment with flosequinan. Am J Cardiol 2002:90:969-73.

43 McDonagh TA, Robb SD, Murdoch DR, et al. Biochemical detection of leftventricular systolic dysfunction. Lancet 1998;351:9-13.

44 Troughton RW, Frampton CM, Yandle TG, et al. Treatment of heart failure guided by plasma aminoterminal brain natriuretic peptide (N-BNP) concentrations. Lancet 2000;355:1126-30.

45 Lemos JA, McGuire DK, Drazner MH. B-type natriuretic peptide in cardiovascular disease. Lancet 2003;362:316-22.

46 Mann DL. Mechanisms and models in heart failure. A combinatorial approach. Circulation 1999;100:999-1008.

47 Baig MK, Mahon N, McKenna WJ, et al. The pathophysiology of advanced heart failure. Am Heart J 1998;135:S216-30.

48 McMurray J, Pfeffer MA. New therapeutic options in congestive heart failure: part I. Circulation 2002:105:2099-106.

49 Anand IS, Florea VG, Fisher L. Surrogate end points in heart failure. J Am Coll Cardiol 2002;39:1414-21 